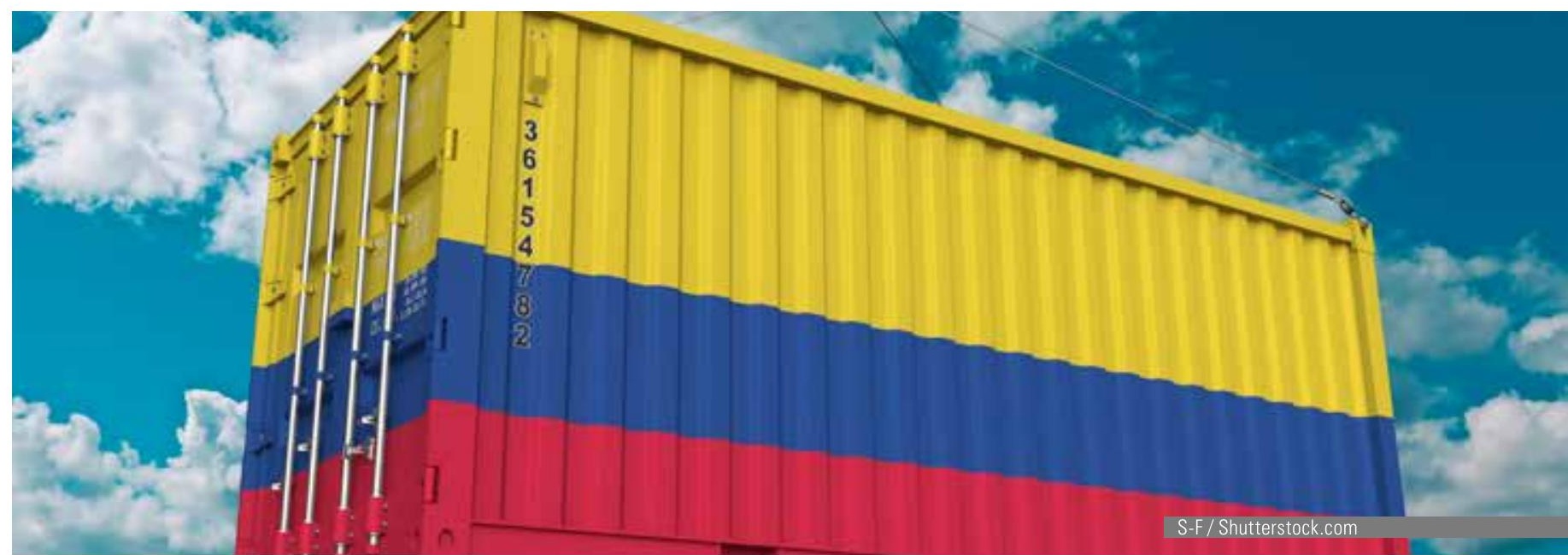

\title{
Estudio de caso sobre la admisibilidad del aguacate Hass colombiano en el mercado estadounidense: oportunidades en el Este de Asia
}

\section{Case Study on the Eligibility of Colombian Hass Avocado in the US Market: Opportunities in East Asia}

>> Juan Carlos Díaz Vasquez

Universidad EAFIT, Colombia

jdiazva@eafit.edu.co

https://orcid.org/0000-0001-9251-5500

\section{Carolina Ardila López}

Universidad EAFIT, Colombia

cardilal@eafit.edu.co

https://orcid.org/0000-0003-4112-2779

Maria Alexandra Guerra Aranguren

Universidad EAFIT, Colombia

mguerra3@eafit.edu.co

Recibido: 26 de marzo 2019

Aprobado: 25 de abril 2019

Publicado: 28 de junio 2019

\section{Resumen}

El presente estudio de caso presenta, de manera sucinta, eventos, circunstancias y actores que participaron en la entrada oficial del aguacate Hass colombiano al mercado estadounidense. El caso expone las características del mercado global del aguacate Hass y los desafíos que Colombia enfrenta para cumplir con la normatividad norteamericana en cuanto a la admisibilidad e importación de frutas a su territorio, además de las oportunidades del mismo en los mercados del Este de Asia. Todas las fuentes examinadas, y el análisis académico conjuntamente realizado en las áreas de negocios internacionales y de comercio exterior, contribuyeron a la construcción del caso. La estructura del artículo responde a las características de escritura propias de los estudios de caso para análisis en clase. 


\section{Palabras clave}

Internacionalización, aguacate Hass, admisibilidad, Estados Unidos, CoIombia, Latinoamérica, agronegocios, barreras no arancelarias, Este de Asia, comercio internacional.

\section{Abstract}

This case study describes the events, circumstances, and actors involved in the official certification of Colombian Hass avocado for exportation to the US market. The research presents the characteristics of the global Hass avocado market and the challenges Colombia faces in order to comply with North American regulations regarding admissibility and importation of fruits into its territory; finally, it discusses the opportunities for this product in the East Asian markets. This article is the product of a joint academic analysis in the areas of international business and foreign trade. The structure of the article follows the writing characteristics of case studies for class analysis.

\section{Key words}

Company internationalization, Hass avocado, eligibility, United States, Colombia, Latin America, agribusiness, non-tariff barriers, East Asia, international trade.

\section{Introducción}

Durante las últimas tres décadas Colombia ha experimentado procesos de tipo político, económico, comercial y normativo hacia la apertura de su economía al ámbito internacional. Uno de los propósitos claros ha sido el aumento y diversificación de las exportaciones colombianas, aspecto en el cual el Gobierno ha aunado esfuerzos para identificar sectores y productos potenciales de la economía, como lo demuestra el caso del "Aguacate, el oro verde de la economía colombiana"(Dinero, 2017), que empieza a convertirse en pilar de desarrollo económico y social del país.

El presente estudio de caso reúne los eventos, circunstancias y actores que han participado en mayor y menor medida en la entrada oficial del aguacate Hass colombiano al mercado estadounidense y sus proyecciones hacia el Este de Asia, región con gran potencial para este tipo de productos y conformada por los países que integran la ASEAN (Association of Southeast Asian Nations): Indonesia, Filipinas, Malasia, Singapur, Tailandia, Vietnam, Brunei, Camboya, Laos, Myanmar; junto con Japón, China y Corea del Sur, de los cuales se ahondará un poco más en cifras en el presente artículo. Esta exposición de temas, lugares y personas busca principalmente servir como ejemplo de la tenacidad y el esfuerzo cooperado entre el sector público y privado para obtener resultados positivos en la proyección internacional de la economía colombiana. Al mismo tiempo, se propone la discusión en torno a las circunstancias específicas de un camino, que ha sido recorrido de manera consistente para llegar al objetivo alcanzado.

El caso, además, expone las características del mercado global del aguacate Hass y los desafíos que Colombia enfrenta en materia de desarrollo de infraestructura y gestión para cumplir con la normatividad norteamericana en cuanto a la admisibilidad e importación de frutas a su territorio, explicando a su vez las oportunidades que se presentan de cara al Este de Asia.

De esta manera, el estudio muestra cómo, con la admisibilidad del producto a este importante mercado, se pone a prueba la capacidad operativa no solo del Gobierno, sino también de todas las personas, empresas, asociaciones e instituciones colombianas que están involucradas en este esfuerzo común. Esto implica cumplir con las exigencias técnicas, sanitarias y fitosani- 
tarias que requiere el bien protegido mercado americano, así como refinar y aumentar la producción nacional, destacando las particularidades del aguacate Hass cultivado en Colombia.

La estructura del caso responde a las características de escritura propias de los estudios de caso para análisis en clase. De acuerdo con esta estructura, se presentan las evidencias del caso apuntando a comprender mejor una decisión tomada (Ellet, 2007). De igual forma, se presentan razones asociadas a los eventos y decisiones que forman el caso en cuestión. Posterior al análisis de la decisión, se muestran planes de acción que sirvan como elemento generador de discusión entre el público objetivo.

\section{Justificación}

La admisibilidad del aguacate Hass colombiano en el mercado estadounidense marca un hito muy importante para la consolidación de Colombia en la economía global. Existen condiciones que hacen de este caso uno particularmente importante de analizar. En primer lugar, considerando la perspectiva nacional, se deben tener en cuenta las circunstancias macro de una economía y de un país que ha pasado por momentos determinantes durante los últimos 30 años. Desde la apertura de la economía a mercados internacionales a comienzos de los noventas, hasta la firma de la paz en $2017^{1}$, Colombia ha venido trabajando arduamente en la consecución y firma de tratados comerciales internacionales que beneficien los productos y servicios nacionales a la hora de entrar a mercados externos.

En segundo lugar, desde la perspectiva internacional, se puede observar cómo otros países productores han desarrollado la infraestructura y la capacidad de gestión en sus diferentes territorios para ser hoy importantes exportadores del aguacate Hass a diferentes lugares del mundo. El caso de México, como primer exportador en la actualidad, es referencia en este asunto. De igual forma, otros países latinoamericanos como República Dominicana, Perú y Chile llevan una considerable ventaja en la producción y exportación de aguacate.

En tercer lugar, el Tratado de Libre Comercio (TLC) con Estados Unidos representa una gran plataforma de favorecimiento arancelario a los productos colombianos. Este Acuerdo comenzó a desarrollarse en el 2003 y, luego de los procesos propios de ajuste legislativo regulatorio y político, llegó a su culmen con la publicación del Decreto 993 de 2012. Sin embargo, el gobierno colombiano ha venido trabajando a través de otras instituciones en allanar el camino para la implementación de este importante acuerdo. Uno de ellos ha sido el Instituto Colombiano Agropecuario (ICA), cuya participación en este caso de estudio ha sido clave para establecer inicialmente los aspectos técnicos necesarios para la producción masiva del aguacate cultivado en el país y para dar a conocer las condiciones específicas del suelo, los climas y la producción de esta fruta en el territorio nacional. De igual forma, empresas productoras, así como distribuidoras internacionales y asociaciones relacionadas con el ramo, juegan un rol decisivo en la apertura del mercado norteamericano y los mercados del Este de Asia para el aguacate Hass colombiano.

\section{Alcance}

Este estudio apunta a la exposición y análisis de las circunstancias específicas que han rodeado la admisibilidad del aguacate Hass al mercado estadounidense y que, por su importancia, lo hacen merecedor de una especial observación. De la misma manera, se muestra cómo todo este proceso de admisibilidad con sus aprendizajes, allanarían el camino para el ingreso del aguacate Hass colombiano en los mercados del Este de Asia. 
El público objetivo de este caso son principalmente los estudiantes de áreas relacionadas con la administración, los negocios internacionales, la internacionalización de la economía colombiana y demás áreas de las ciencias sociales. Igualmente, el estudio está dirigido a todas las personas, grupos de interés e instituciones que directa o indirectamente participan del negocio del aguacate Hass en Colombia y quieren ampliar su conocimiento sobre el tema. Finalmente, cabe destacar que este caso colombiano puede servir como parte de los esfuerzos para construir un país más consciente de la riqueza que alberga el campo, cuyo brillo fue durante muchos años opacado por las duras condiciones de un conflicto armado largo y doloroso para todos los colombianos.

\section{Metodología}

El presente estudio de caso se elaboró con la información de fuentes primarias y secundarias disponibles a la fecha. Entre las fuentes primarias fueron entrevistados funcionarios del gobierno colombiano, entre ellos el director de la Oficina Comercial del Gobierno de Colombia en Estados Unidos, el representante de ProColombia en Washington D.C. para los asuntos relacionados con el agro, así como algunos empresarios y funcionarios gremiales.

Las fuentes secundarias se componen de artículos consultados en medios públicos y asociaciones privadas, así como de estudios de las cámaras de comercio y gremios de exportadores, tanto de Colombia como de los demás países estudiados.

Estas fuentes examinadas, y el análisis académico conjuntamente realizado en las áreas de negocios internacionales y de comercio exterior, contribuyeron a la construcción del caso y sus notas pedagógicas.

\section{El mercado mundial del aguacate Hass}

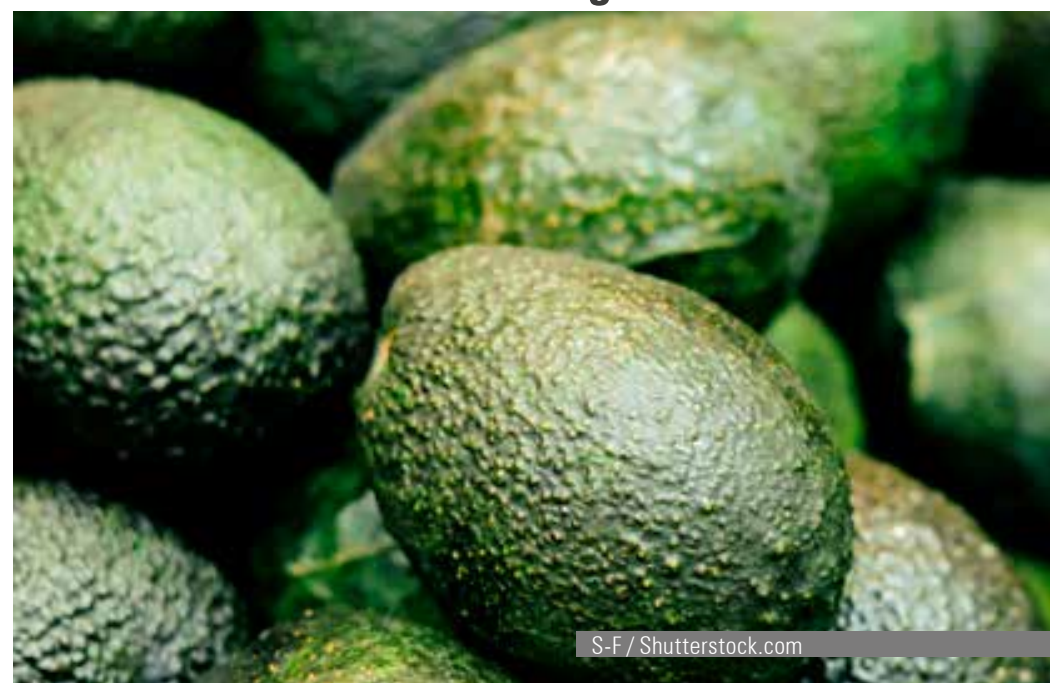

Entre 2014 y 2015 las exportaciones colombianas de aguacate crecieron 188\%, al pasar de USD 3,57 millones a USD 10,1 millones, y se estima que el consumo de aguacate tendrá un aumento de 10\% a 15\% en los próximos años (Minagricultura, 2016).

Además de Estados Unidos, país que lidera la lista de los importadores del producto con cerca de USD 2.000 millones comprados en el 2016 (Mincit, 2017), figuran principalmente Países Bajos, Francia, Reino Unido, España, Canadá y Japón. 
El mercado del aguacate europeo, que en 2011 era de 186 mil toneladas, cerró 2015 con 343 mil toneladas (Neederlands Ministry of Foreign Affairs, 2016), entre las que se destacan el aguacate Hass, pero también se venden otras variedades en menor proporción. Las más altas tasas de consumo se registran en España, Francia, Alemania y el Reino Unido, siendo los Países Bajos, además de un importante consumidor, el principal punto de acceso del aguacate a Europa, con una participación de 47\% de las importaciones de aguacate (Neederlands Ministry of Foreign Affairs, 2016). Debido a que la demanda crece más deprisa que la velocidad de abastecimiento, se han elevado sus precios y en particular, el producto ready-to-eat o listo para consumir, está aumentando en dicha región (Simfruit, 2016).

\section{Figura 1. Principales importadores europeos de aguacate fresco}

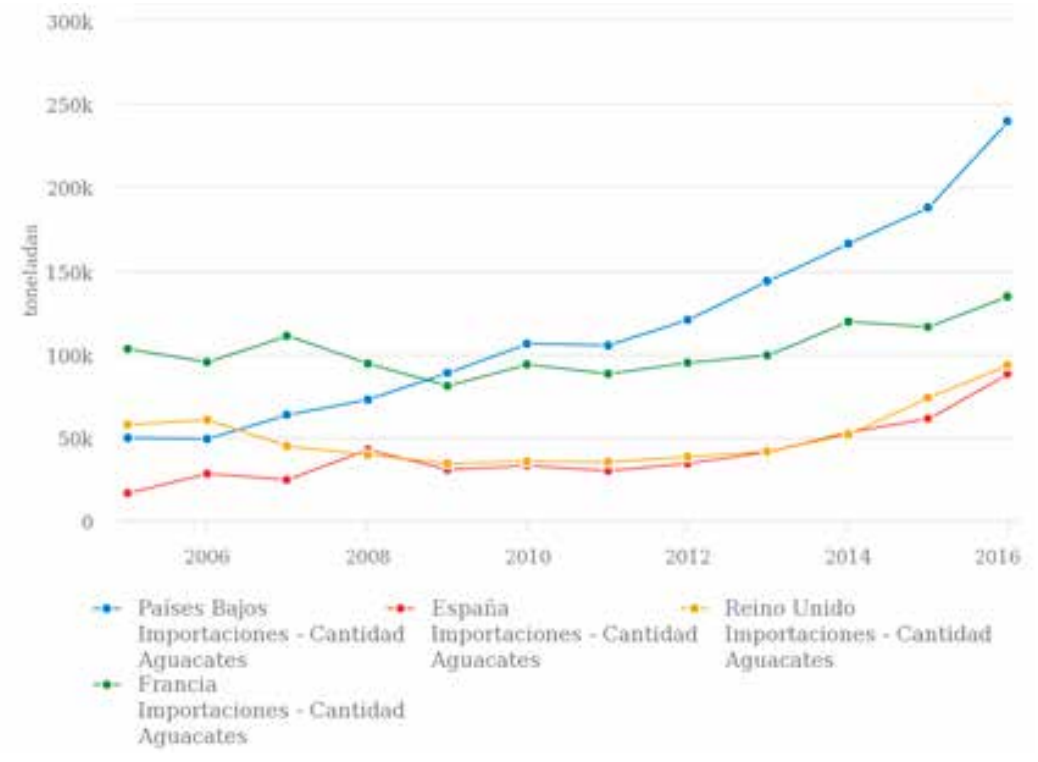

Fuente: FAOSTAT (2019)

El mercado estadounidense de aguacate es tres veces mayor que el europeo y cerca de diez veces mayor que el del este asiático (Trade Map, 2019). 96\% de la demanda se refiere a la variedad Hass (Mincit, 2017). Aun cuando Estados Unidos produce unas 200 mil toneladas anuales, debió importar en 2016 cerca de 860 mil toneladas (Analdex, 2017) para abastecer su consumo interno, que se duplicó entre 2007 y 2016 (IndexBox, 2017). México es el principal origen del aguacate Hass que importa Estados Unidos. 


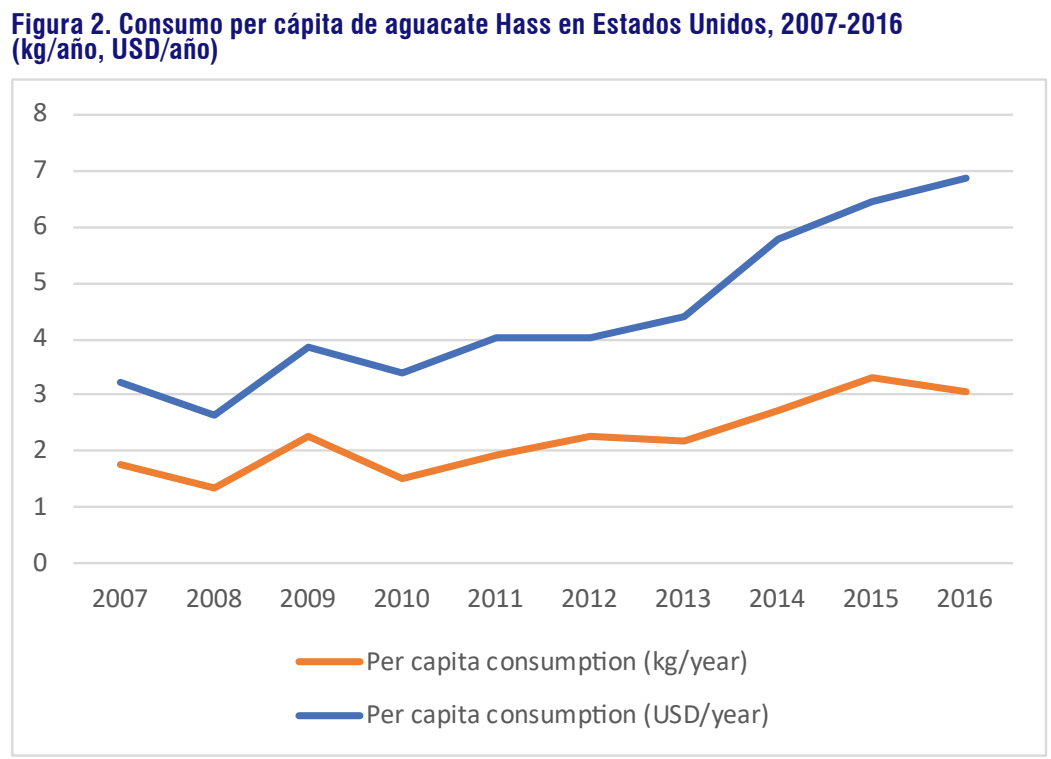

Fuente: IndexBox (2017)

El mercado aumenta por el crecimiento de la población hispana, por la tendencia a los alimentos étnicos, saludables y por la labor de promoción (IndexBox, 2017). México ha jugado un papel clave en todo este proceso de crecimiento, siendo consistente con su abastecimiento sin comprometer la calidad del producto. De los demás países latinoamericanos, Colombia también tiene el potencial de tener un abastecimiento estable del producto a Estados Unidos, beneficiándose además de su cercanía geográfica.

México también encabeza la lista de proveedores de aguacate Hass en Japón, en los países de la Asociación de Naciones del Sureste de Asia (ASEAN) y la República de Corea, también denominada como Corea del Sur (Trade Map, 2019).

Tabla 1. Cifras del aguacate Hass exportado por México, Chile y Perú al Este de Asia (miles

Cifras del aguacate Hass exportado por México, Chile y Perú al Este de Asia (USD millones)

\begin{tabular}{|c|c|c|c|c|}
\hline \multicolumn{5}{|c|}{$\begin{array}{c}\text { Exportaciones de aguacate Hass mexicano al Este de Asia } \\
\text { Producto: 08044001 Paltas (aguacates): aguacates (paltas). } \\
\text { Unidad: USD millones }\end{array}$} \\
\hline Destino & 2014 & 2015 & 2016 & 2017 \\
\hline ASEAN* & 0,1 & 0,1 & 2,7 & 5,8 \\
\hline Japón & 112,3 & 104,8 & 161,5 & 181,5 \\
\hline China & 8,3 & 24 & 24,1 & 24,7 \\
\hline Hong Kong & 1,6 & 2,2 & 5,1 & 7,3 \\
\hline Corea del Sur & & 0,5 & 1 & 5,5 \\
\hline
\end{tabular}




\begin{tabular}{|c|c|c|c|c|}
\hline \multicolumn{5}{|c|}{$\begin{array}{c}\text { Exportaciones de aguacate Hass chileno al Este de Asia } \\
\text { Producto: } 080440 \text { Aguacates frescos o secos } \\
\text { Unidad: USD millones }\end{array}$} \\
\hline Destino & 2014 & 2015 & 2016 & 2017 \\
\hline \multicolumn{5}{|l|}{ ASEAN* } \\
\hline China & 0,1 & 7,2 & 32,7 & 51,7 \\
\hline $\begin{array}{l}\text { Hong Kong, } \\
\text { China }\end{array}$ & 2,5 & 1,4 & 0,7 & 1,7 \\
\hline Japón & 2 & 0 & 1 & 0 \\
\hline Corea del Sur & & & & \\
\hline
\end{tabular}

\begin{tabular}{|c|c|c|c|c|}
\hline \multicolumn{5}{|c|}{$\begin{array}{c}\text { Exportaciones de aguacate Hass peruano al Este de Asia } \\
\text { Producto: } 0804400000 \text { Aguacates "paltas", frescos o secos } \\
\text { Unidad: USD milones }\end{array}$} \\
\hline Destino & 2014 & 2015 & 2016 & 2017 \\
\hline \multicolumn{5}{|l|}{ ASEAN* } \\
\hline China & 0,2 & 0.0 & 2,1 & 9,2 \\
\hline \multicolumn{5}{|l|}{$\begin{array}{l}\text { Hong Kong, } \\
\text { China }\end{array}$} \\
\hline Japón & 2,1 & 1,0 & 4,6 & 5,4 \\
\hline Corea del St & & & & \\
\hline
\end{tabular}

Tablas elaboradas por los autores con información de Trade Map (2019) * Datos de ASEAN basados en las importaciones registradas

\section{Características de la producción global de Aguacate Hass}

El aguacate Hass, originario de Latinoamérica y patentado por el estadounidense Rudolph Hass en 1935, fue introducido globalmente en el mercado en 1960 y es hoy la variedad de aguacate más cultivada en el mundo (Comité de paltas Hass de Chile, 2015)². Su prolongada estación de cosecha y beneficios comprobados para la salud, han permitido aumentar el consumo mundial de la fruta en los últimos años.

Entre los países latinoamericanos que figuran en el comercio mundial del aguacate, Hass además de México, están República Dominicana, Perú, Colombia y Chile (Analdex, 2017). El producto también se cultiva en varios países de África, el sur de Asia, España, Nueva Zelanda e Israel. 
Figura 3. Principales productores de aguacate en el mundo 2016 (Toneladas)

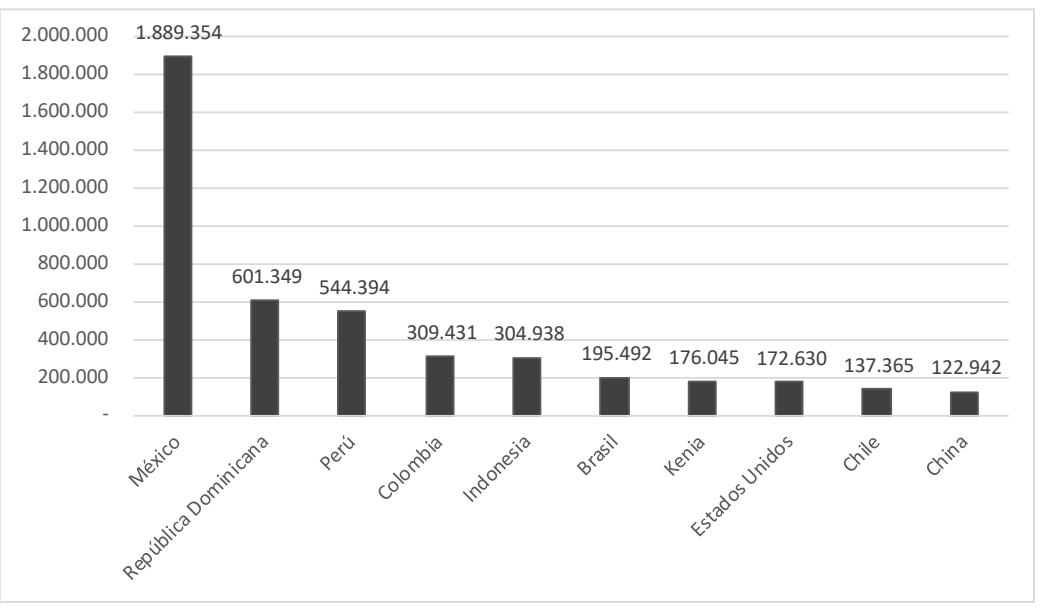

Fuente: FAOSTAT (2016)

A continuación, se describen las características de producción de los países latinoamericanos líderes en el mercado.

\subsection{México}

México, país que exporta más de 1'400.000 toneladas (El Financiero, 2017b), es hoy el mayor proveedor de la variedad Hass, al estimarse que, de cada 10 aguacates comercializados a nivel mundial, tres son vendidos por productores mexicanos, resaltando además que 90\% del aguacate Hass que se importa en Estados Unidos proviene de dicho país (El Financiero, 2017a). Pero esto no es un hecho que se dio de la noche a la mañana, responde a los grandes esfuerzos de mejora, y arduas negociaciones que los cultivadores y el gobierno mexicano llevaron a cabo durante la década de 1990 con los organismos de control estadounidense, para que el producto, que ya se comercializaba en Estados Unidos a principios del siglo XX (Orden \& Peterson, 2003), tuviera una entrada definitiva a dicho mercado. Los mexicanos lograron su propósito de manera ejemplar, cumpliendo con las rigurosas barreras técnicas impuestas por el gobierno estadounidense y abriéndose camino entre las fuertes presiones de los cultivadores de California quienes se opusieron con firmeza al ingreso de un producto que, antes que amenazar su industria, la catapultó a los niveles que hoy se conocen y de los cuales se están beneficiando todos los países productores. A diferencia de Colombia y Perú que solo pueden ingresar el producto al mercado estadounidense a través de sus puertos continentales, México hoy tiene acceso además a Hawái y Puerto Rico (USDA, 2017).

\subsection{Chile}

Cerca de 30.000 toneladas de aguacate Hass vende Chile a Estados Unidos (Comité de paltas Hass de Chile, 2018), cifra muy inferior a las 1'400.000 toneladas anteriormente mencionadas por parte de la competencia mexicana; sin embargo, esto no siempre fue así. El aguacate Hass mexicano, algo similar al caso colombiano que ha logrado el acceso del producto mucho después de firmado el TLC, tuvo su ingreso definitivo al mercado estadounidense algunos años después de entrada en vigencia del NAFTA; antes de eso, Chile y Nueva Zelanda se repartían dicho mercado, en especial, en los meses de agosto a diciembre, cuando baja de manera considerable la co- 
secha californiana (Orden \& Peterson, 2003). El aguacate o "palta" chileno es el segundo producto de exportación más importante del sector frutícola de Chile, y tiene una clientela bastante más diversa que Colombia. Se estima que dicho país tiene 212.000 hectáreas cultivadas, de las cuales 45\% se destina para el consumo interno, luego lo siguen Europa con 25\%, Estados Unidos con aproximadamente $20 \%$, Latinoamérica con $7 \%$ y Asia con $3 \%$ (Comité de paltas Hass de Chile, 2015). Chile logró ingresar en el mercado chino en 2014 y, desde entonces, el volumen de sus exportaciones hacia este destino ha crecido de forma notable, siendo actualmente líder en proveeduría a dicho país, por encima de México (Trademap, 2019).

Tabla 2.

\begin{tabular}{|c|c|c|c|c|}
\hline \multicolumn{5}{|c|}{ Importaciones aguacate hass toneladas } \\
\hline & 2014 & 2015 & 2016 & 2017 \\
\hline China & 4,066 & 15,989 & 25,129 & 32,137 \\
\hline
\end{tabular}

\begin{tabular}{|c|c|c|c|c|}
\hline \multicolumn{5}{|c|}{ Proveedores aguacate hass toneladas a China } \\
\hline & 2014 & 2015 & 2016 & 2017 \\
\hline México & 3,966 & 13,582 & 9,996 & 8,754 \\
\hline Chile & 100 & 2,263 & 11,595 & 16,707 \\
\hline Perú & & 144 & 3,568 & 6,667 \\
\hline
\end{tabular}

Tabla elaborada por los autores con información de Trade Map (2019)

\subsection{Perú}

El aguacate Hass del Perú, el cual tiene poca aceptación por el consumidor local quien prefiere las variedades de piel verde, se destina prácticamente en su totalidad a los mercados internacionales. El incremento en el consumo mundial también ha beneficiado la industria de dicho país, pasando de exportar cerca de 30 mil toneladas en el primer semestre del 2015, a 44 mil en el primer semestre del 2017 (ProHass Perú, 2017). Estados Unidos le otorgaron acceso al aguacate Hass peruano en el año 2010 (Ministerio de Relaciones Exteriores, 2011) y, cuando México no logra llegar a tiempo al mercado estadounidense, es común encontrar aguacate Hass peruano en reemplazo; sin embargo, sus principales mercados de exportación continúan siendo Países Bajos, España y Reino Unido, a los cuales vende desde principios del año 2000. El producto peruano también ha venido ingresando en el Este del Asia, consolidando exportaciones periódicas en especial hacia China y Japón desde su admisibilidad a mediados de la presente década, tal como se aprecia en el Tabla 1.

\subsection{República Dominicana}

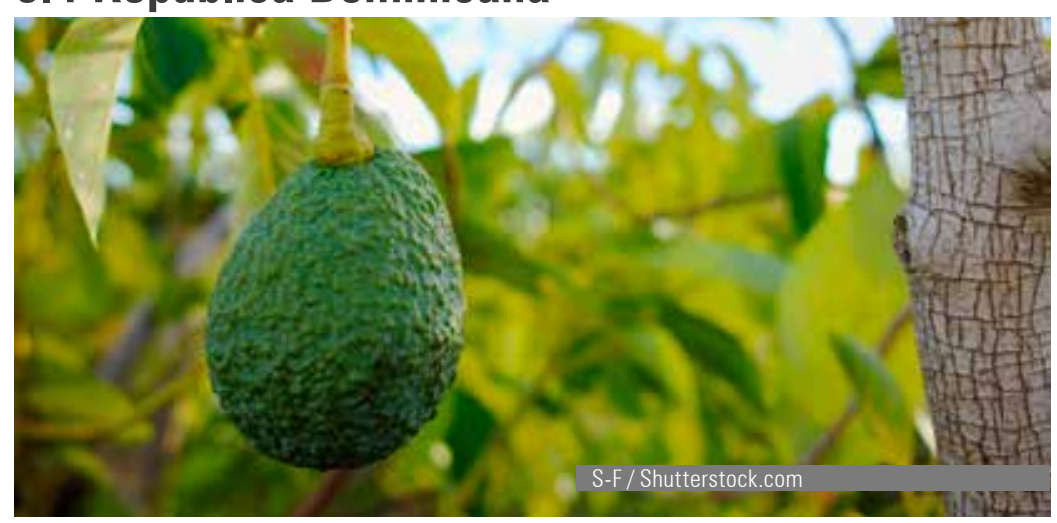


República Dominicana es otro caso en el que el consumidor local prefiere aguacate de piel verde, lo que hace que solo $15 \%$ de sus 21 mil hectáreas (Rudert, 2017) y 19 variedades cultivadas (Freshplaza, 2015) sean Hass. Esta isla, que viene recuperando su ritmo comercial después de que se detectara en sus productos la presencia de la mosca de la fruta mediterránea en 2015, posee una ventaja que dinamiza enormemente su industria y es el tiempo de llegada a los mercados internacionales. Según el exportador dominicano César Pérez (citado en Freshplaza, 2015)

Los exportadores se benefician de los ocho aeropuertos internacionales de la República Dominicana. De esa forma es posible tener los productos en sus destinos en cualquier parte del mundo en menos de 15 horas. Si se usa transporte marítimo solo se tarda tres días en llegar a Florida (EE. UU.), nueve días para enviar los productos al puerto de Róterdam y al Reino Unido. (párr. 3)

A pesar de las limitaciones para expandir el cultivo internamente, República Dominicana figura actualmente como el segundo mayor productor de aguacate en el mundo, después de México.

\subsection{Producción en Colombia}

El quinto productor mundial de aguacate de todos los tipos es Colombia, pero aún no figura entre los grandes exportadores de este producto (Analdex, 2017). Solo hasta mayo de 2010 fue posible la primera exportación de la fruta en un contenedor de 20 toneladas con destino a Francia (AmCham Colombia, 2015); pero ese mismo año el precio cayó fuertemente, incluso por debajo de los costos de producción, y muchos cultivadores desistieron, como lo afirma Ricardo Uribe, gerente general de Cartama (citado en Dinero, 2017), empresa líder en la comercialización del aguacate Hass colombiano.

En 2010, cuando ya nos sentíamos listos para exportar, el precio cayó fuertemente, incluso por debajo de los costos de producción y muchos otros cultivadores sacaron la mano, pues producir aguacate de manera tecnificada es bastante costoso. Pese a que la curva de aprendizaje estaba muy dura, persistimos en nuestra aspiración de exportar, así que nos certificamos y, aunque inicialmente le vendíamos a una comercializadora internacional, desde 2015 lo hacemos nosotros directamente.

Colombia, como los demás países latinoamericanos, se ha beneficiado del incremento del consumo global mejorando exponencialmente el propio desempeño productivo y comercial, tanto que hoy el aguacate es el sexto producto agrícola de exportación, después del café, el banano, las flores, el aceite de palma y el azúcar (Dinero, 2017). En el aguacate Hass están las apuestas y es por ello que, según las estadísticas de la Asociación Hortifrutícola de Colombia (Asohofrucol), al cierre de 2016 se produjeron 78.547 toneladas de aguacate Hass en un área sembrada que llega aproximadamente a 14.084 hectáreas (Asohofrucol, s.f.).

Los departamentos con mayor potencial de producción son Antioquia, Caldas y Tolima, pero se encuentran cultivos en otras regiones gracias a que Colombia goza de un clima ideal y de los diversos pisos térmicos en los que el cultivo puede desarrollarse (Dinero, 2017). Este factor representa una ventaja competitiva para llegar a los mercados internacionales, prácticamente durante todo el año con cosecha permanente (Pavas, 2015), si se controlan todos los aspectos relacionados con una óptima producción. 
Tabla 3. Producción de aguacate por departamentos en Colombia 2014 y 2015 (HA)

\begin{tabular}{|c|c|c|c|c|}
\hline Departamentos & Área (Ha) 2014 & Área (Ha) 2015 & $\begin{array}{c}\text { Producción } \\
\mathbf{2 0 1 4}\end{array}$ & $\begin{array}{c}\text { Producción } \\
\mathbf{2 0 1 5}\end{array}$ \\
\hline Tolima & 8.088 & 9.291 & 65.945 & 60.718 \\
\hline Antioquia & 4.784 & 5.077 & 47.821 & 48.427 \\
\hline Caldas & 3.848 & 4.255 & 43.190 & 40.268 \\
\hline Santander & 3.068 & 3.101 & 26.742 & 19.716 \\
\hline Bolívar & 3.097 & 3.021 & 32.293 & 30.527 \\
\hline Cesar & 2.018 & 2.342 & 16.153 & 19.138 \\
\hline Valle del Cauca & 1.643 & 1.848 & 24.090 & 25.685 \\
\hline Ouindío & 1.205 & 1.692 & 10.228 & 13.246 \\
\hline Risaralda & 1.390 & 1.154 & 16.808 & 14.091 \\
\hline Total & $\mathbf{2 9 . 1 4 4}$ & $\mathbf{3 1 . 7 8 1}$ & $\mathbf{2 8 3 . 2 7 3}$ & $\mathbf{2 7 1 . 8 1 6}$ \\
\hline
\end{tabular}

Fuente: Dinero $(2017)^{1}$

Tabla 4. Meses de cosecha del aguacate Hass en Colombia por región

\begin{tabular}{|c|c|c|c|c|c|c|c|c|c|c|c|c|}
\hline ZONA & ENE & FEB & MAR & ABR & MAY & JUN & JUL & AGO & SEP & ОСТ & NOV & DIC \\
\hline ORIENTE-ANTIOQUIA & \multicolumn{5}{|c|}{ HASS } & & & & & & & HASS \\
\hline NORTE-TOLIMA & HASS & & & & \multicolumn{2}{|c|}{ HASS } & & & & \multicolumn{3}{|c|}{ HASS } \\
\hline EJE CAFETERO & \multicolumn{2}{|c|}{ HASS } & & & & & & & & \multicolumn{3}{|c|}{ HASS } \\
\hline SUROESTE-ANTIOQUIA & HASS & & & & & & & & & \multicolumn{3}{|c|}{ HASS } \\
\hline MESETA POPAYÁN & & & & & & & & & \multicolumn{4}{|c|}{ HASS } \\
\hline NORTE DEL VALLE & HASS & & & & & & & & \multicolumn{4}{|c|}{ HASS } \\
\hline
\end{tabular}

Fuente: Pavas (2015)

\subsection{Exportaciones Colombianas}

Mientras en 2011 se exportaron al mundo USD 171.000 en aguacates colombianos, en 2015 las ventas llegaron a USD 10,5 millones y en 2016 se exportaron USD 35 millones, equivalentes a 18.200 toneladas. España, Países Bajos, Francia y Reino Unido son los principales destinos de exportación del aguacate Hass colombiano: en 2016 cerca de 7.000 toneladas fueron importadas por Países Bajos, lo que representa 42\% del total de lo exportado a Europa en aguacate Hass colombiano (Mincit, 2017). El país posee una ventaja en tiempos frente a la competencia latinoamericana, pues las exportaciones de Chile y Perú se demoran entre 23 y 25 días para llegar a Europa, mientras que a las de Colombia solo les toma 11 días (Dinero, 2017). Colombia también exporta buena cantidad de aguacate a Centroamérica y a algunas islas del Caribe, y ha venido haciendo los primeros despachos al Este del Asia llegando exitosamente a Hong Kong en 2017, lo que representó USD 48 mil dólares en exportaciones (Trade map, 2019).

Evaluaciones Agropecuarias Municipales. Oficina de Planeación y Prospectiva -Grupo de información y estadísticas sectoriales. Minagricultura. 
Tabla 5. Exportaciones colombianas de aguacate Hass. 2014 y 2015(USD y Kg)

\begin{tabular}{|c|c|c|c|c|c|c|c|c|}
\hline $\begin{array}{c}\text { Principales } \\
\text { Países }\end{array}$ & $\begin{array}{c}\text { Valor FOB } \\
\text { USD 2014 }\end{array}$ & $\begin{array}{c}\text { Valor FOB } \\
\text { USD 2015 }\end{array}$ & Vr. \% & $\begin{array}{c}\text { Part. } \\
\mathbf{2 0 1 5}\end{array}$ & $\begin{array}{c}\text { Kg Netos } \\
\mathbf{2 0 1 4}\end{array}$ & $\begin{array}{c}\text { Kg. Netos } \\
\mathbf{2 0 1 5}\end{array}$ & Vr. \% & $\begin{array}{c}\text { Part } \\
\mathbf{2 0 1 5}\end{array}$ \\
\hline Países Bajos & $2^{\prime} 852.147$ & $6^{\prime} 162.028$ & 116,05 & 59,95 & 1.369 .058 & $3.338,91$ & 143,89 & 60,24 \\
\hline Reino Unido & 457.694 & $2^{\prime} 429.666$ & 430,85 & 23,64 & 223.592 & $1.169,62$ & 423,14 & 21,10 \\
\hline España & 147.321 & 930.458 & 531,58 & 9,05 & 80.370 & 582.509 & 624,78 & 10,51 \\
\hline Francia & 68.542 & 668.935 & 875,95 & 6,51 & 43.998 & 407.334 & 825,80 & 7,35 \\
\hline Panamá & 30.620 & 56.687 & 85,13 & 0,55 & 17.160 & 21.894 & 27,59 & 0,39 \\
\hline Martinica & $*$ & 16.266 & $*$ & 0,16 & $*$ & 9.845 & $*$ & 0,18 \\
\hline Guayana Fr & $*$ & 12.390 & $*$ & 0,12 & $*$ & 10.915 & $*$ & 0,20 \\
\hline Aruba & 2.315 & 680 & $-70,64$ & 0,01 & 4.246 & 785 & $-81,51$ & 0,01 \\
\hline Curazao & $*$ & 589 & $*$ & 0,01 & $*$ & 411 & $*$ & 0,01 \\
\hline Italia & 295 & 556 & 88,38 & 0,01 & 108 & 174 & 61,11 & 0,0031 \\
\hline Total & $33^{\prime} 573.365$ & $10^{\prime} 279.279$ & 187,66 & 100 & 1.759 .581 & 5.542 .90 & 215,02 & 100 \\
\hline
\end{tabular}

Fuente: Analdex (2016) ${ }^{2}$

La meta del gobierno colombiano era que, con la reciente posibilidad de ingreso al mercado norteamericano, en 2018 las exportaciones de aguacate fueran del orden de USD 100 millones (Minagricultura, 2016); un incremento de casi tres veces el monto exportado en 2017. Sin embargo, el crecimiento fue menor, ya que en 2017 el país le vendió al mundo USD 52,9 millones, y en los 10 primeros meses del 2018 las ventas superaron los USD 53,6 millones. Al comparar este monto con enero-octubre del 2017, la variación positiva fue de 32,6\% (Mincit, 2019).

\section{Instituciones que apoyan la industria del aguacate Hass en Colombia}

El aguacate Hass colombiano, como lo hemos comentado a lo largo del caso, es el producto agrícola del momento. Debido a su rendimiento de cultivo por hectárea, está por encima de grandes competidores internacional y, hoy en día, es un producto simbólico del agro colombiano que ha conquistado el mercado europeo por su calidad, sabor y, además, por cumplir con los requerimientos sanitarios. Las medidas sanitarias son precisamente las que habían limitado por años su acceso al mercado estadounidense ya que, bajo las condiciones climáticas del territorio colombiano, se consideraba que existía un mayor riesgo de propagación de plagas propias de esta fruta. No obstante, ante este panorama, el gobierno colombiano, bajo liderazgos del Ministerio de Agricultura y el Instituto Colombiano Agropecuario (ICA), emprendió un arduo trabajo, junto con los cultivadores, para lograr la erradicación y prevención de riesgos sanitarios, lo cual redundó en la reciente admisibilidad del aguacate Hass al mercado norteamericano. 
Como lo menciona Carlos Alberto Soto, Subgerente de Protección Vegetal del ICA: "Se intenta que tanto pequeños como grandes productores tengan un manejo integral y empresarial de los predios, implementar buenas prácticas agrícolas, un minucioso y continuo monitoreo de los cultivos para detectar y controlar las plagas"; tales fueron los objetivos de esta entidad con las capacitaciones ofrecidas a los productores para lograr la admisibilidad en el mercado estadounidense.

Así mismo, se indican las plagas a controlar:

Para el cultivo de aguacate se definieron a las especies Heilipus lauri Boheman, Heilipus trifasciatus Fabricius (Coleoptera: Curculionidae) y Stenoma catenifer Walsingham (Lepidoptera: Elachistidae), más conocidas como barrenadores del fruto, como plagas cuarentenarias y que restringen el comercio de este producto hacia los mercados internacionales (Agronet, 2015).

Antioquia, departamento ubicado al noroccidente de Colombia y que cuenta con uno de los periodos más largos de cosecha de aguacate Hass en el país, adelanta desde su Gobernación el macroproyecto Desarrollo tecnológico, productivo y comercial del aguacate en Antioquia (Corpoica, 2017). Esta es una investigación multidisciplinaria que se hace desde la Secretaría de Agricultura y Desarrollo, liderada por la Corporación Colombiana de Investigación Agropecuaria (Corpoica). La investigación cubre actividades en los campos de agrobiodiversidad, ecofisiología, Manejo Integrado de Plagas (MIP), índices de cosecha y poscosecha y zonificación.

Dicho proyecto se desarrolla a lo largo de la cadena productiva desde sus inicios, para garantizar el cumplimiento de los estándares del mercado internacional y así obtener el objetivo de posicionarlo como el cuarto producto de exportación del agro colombiano.

La Asociación Nacional de Exportadores (Analdex), creada con el propósito de promover y apoyar a las empresas exportadoras, reportó una variación positiva para el aguacate Hass en Colombia de 228\% en volumen para 2016, representado en 18.201 toneladas, comparadas con las 5.543 del año anterior (Corpoica, 2017).

El gobierno de Juan Manuel Santos, que culminó en 2018, priorizó desde 2015 el aguacate Hass como un Proyecto de Interés Nacional Estratégico (PINE) en el que se articulan diferentes entes estatales como los ministerios de Comercio Industria y Turismo, Agricultura, Relaciones Exteriores, y otros como el Instituto Colombiano Agropecuario (ICA), ProColombia y el Programa de Transformación Productiva (PTP). De manera directa, se involucran productores, exportadores, Analdex y la Corporación de Productores y Exportadores de Colombia —CorpoHass— (Minagricultura, 2016). El interés inicial del gobierno fue cultivar aguacate Hass en los Montes de María, zona ubicada entre los departamentos de Sucre y Bolívar, para sustituir los cultivos ilícitos allí presentes; sin embargo, posteriormente se identificó que dicha zona no era apta para el cultivo de la variedad Hass, esfuerzo político que pudo haber sido aprovechado en otras zonas del país (Claudia Candela, 2017).

CorpoHass, tiene en sus manos la defensa de los intereses de los productores de aguacate tanto en el mercado interno como externo, y fue creado como uno de los requisitos exigidos por el gobierno de Estados Unidos a través del Animal and Plant Health Inspection Service (APHIS, por sus siglas en inglés). El ICA, mediante la Subgerencia de Regulación Sanitaria y Fitosanitaria, trabajó desde 2011 en la recolección de información para adelantar el proceso de cumplimiento del control sanitario (ICA, 2013).

En septiembre 13 de 2017 se lanzó el Proyecto Asistencia técnica gremial para fortalecer la admisibilidad del aguacate Hass colombiano al mercado de Estados Unidos por parte de Asohofrucol, entidad que desde 1995 representa los intereses de cultivadores de frutas y hortalizas, y quien además se 
encarga de administrar el Fondo Nacional de Fomento Hortofrutícola (FNFH), para la inversión de proyectos productivos en el país. El proyecto cuenta con recursos otorgados por el Ministerio de Agricultura y es administrado por el Ministerio de Comercio, Industria y Turismo, a través del PTP. Está prevista su ejecución por parte de Asohofrucol y Analdex con acompañamiento técnico del ICA, especialmente en el tema de plagas cuarentenarias, para beneficio de productores en los Departamentos de Tolima, Antioquia, Caldas, Valle del Cauca, Quindío y Cauca (Asohofrucol, 2017).

\section{Contexto de admisibilidad del aguacate Hass en Estados Unidos}

Aun con la firma de acuerdos comerciales que eliminan las barreras de tipo arancelario, Estados Unidos, a través del APHIS —entidad que vigila la salud animal y vegetal-, ha implementado una serie de condiciones de tipo no arancelario que limitan la entrada de fruta y verdura a dicho país. Las restricciones para el aguacate Hass y otros cultivos, se presentan a manera de medidas sanitarias y fitosanitarias, creadas con el propósito de proteger la salud y la conservación de las especies y el medio ambiente, antes que limitar el comercio (Jouanjean, Maur \& Shepherd, 2016); sin embargo, lograr cumplir con estas medidas en dicho mercado sugiere un reto muy grande, tanto a nivel técnico, como en dinero y en tiempo para los productores de los mercados emergentes, muchos de los cuales optan por destinos con requisitos más alcanzables.

En el caso de México, el país productor y comercializador de aguacate Hass más importante en la actualidad, hubo dificultad para introducir este producto a Estados Unidos desde 1914, año en que las autoridades estadounidenses descubrieron cultivos de aguacate mexicano contaminados con plagas de importancia cuarentenaria. Después de varios años de probar el control de riesgo frente a esta situación, solo hasta 1997 el APHIS publicó la regla final para su admisibilidad y permitió la importación a unos pocos Estados, de aguacate Hass cultivado en Michoacán, condición que ha sido ampliada a lo largo del tiempo. El país azteca avanzó con la firma del North American Free Trade Agreement (NAFTA, por sus siglas en inglés) y Estados Unidos agilizó los protocolos permitiendo el acceso por Alaska y el norte del país, para evitar así los riesgos.

Las medidas de protección fitosanitaria al aguacate californiano llevaron a los mexicanos a un trabajo juicioso, para demostrar con estudios científicos el cumplimiento de medidas y así concretar su acceso; por su parte, las autoridades norteamericanas crearon en el 2002 el Hass Avocado Board, institución que promueve el consumo de aguacate Hass en Estados Unidos (HAB, 2017). Con esto, México logró ampliar su mercado sin afectar al aguacate de California, de manera que el aguacate Hass tuvo una contribución determinante en el desarrollo del sector hortofrutícola en ese país.

De acuerdo con información del Foreign Agricultural Service (FAS, por sus siglas en inglés) u Oficina de Asuntos Agrícolas del Departamento de Agricultura de Estados Unidos (USDA) se estima que el costo total de cumplir con los estrictos procedimientos fitosanitarios les costaría a los productores y empacadores mexicanos unos USD110.000 anualmente (Bredahl, 2000).

\section{Proceso de admisibilidad del aguacate Hass colombiano a Estados Unidos}

El caso del aguacate mexicano se presenta como un antecedente ejemplar en la admisibilidad del producto al mercado de Estados Unidos. Del acceso 
del aguacate colombiano se había hablado hace 40 o 50 años, cuando aún no había acuerdos sanitarios o las medidas sanitarias todavía eran muy subjetivas.

Mientras México avanzaba en la consolidación del mercado de aguacate Hass en Estados Unidos, Colombia contaba con el apoyo del gobierno norteamericano del que recibía fondos para el manejo de cultivos ilícitos (Plan Colombia), el cual incluyó desde 2002 el programa de sustitución de cultivos que buscaba alternativas viables en zonas productivas. Esto también dio origen al Centro de Excelencia Fitosanitaria (ICA, s.f.) con el que se priorizaron ciertos productos como la uchuva, la papaya, el lulo, la pitahaya, el tomate de árbol, las pasifloras, entre otros.

\subsection{APHIS e ICA}

Según Enrique Millán Mejía (2017), director de la Oficina Comercial del Gobierno de Colombia en Estados Unidos, el proceso de admisibilidad del aguacate Hass colombiano se inició desde hace más de 10 años, cuando el ICA, como representante sanitario de Colombia, envió en el 2005 una carta oficial de requerimiento a su homólogo norteamericano APHIS. En dicho año, en convenio con Asohofrucol, que cuenta con el Fondo Nacional de Fomento Hortifrutícola, se aportaron recursos para levantar el primer paso técnico de gestión de riesgos de plagas, el cual se plasma en el Pest Risk Assessment (PRA) (United States Department of Agriculture, 2017) y con el cual se inicia el proceso técnico de recolección de información.

Con la llegada del Tratado de Libre Comercio entre Colombia y Estados Unidos, se priorizaron de nuevo los productos agrícolas potenciales de exportación y se puso en primer lugar al aguacate Hass; para este entonces, la evaluación de riesgo ya se había adelantado. Dicha evaluación de riesgo, así como las medidas de mitigación, quedaron escritas en la Regla Final, (United States Department of Agriculture, 2017), paso posterior al estudio técnico científico.

Dicho proceso de admisibilidad contó con la intervención del sector público en cabeza del jefe de gobierno e inspectores del Instituto Colombiano Agropecuario (ICA). La academia, de igual forma, ha tomado parte con el apoyo de documentación científica en manejo de riesgo y control de las plagas que afectan los cultivos de exportación de este producto.

El proceso contempló un mecanismo de equivalencia sanitaria compuesta de una fase técnico-científica, luego una regulatoria y, por último, una más operativa donde la dimensión política está presente de manera transversal en cada una de las fases. La fase técnico-científica fue la de mayor complejidad, duró varios años y en ella se evaluaron todos los riesgos fitosanitarios asociados al producto en origen y los efectos en el mercado de destino. En esta etapa de validación hubo intercambio de conocimiento y opiniones de expertos en el campo de la entomología y fitopatología (Millan y Forero, 2017).

\subsection{Fase técnico-científica}

Dentro de las medidas de mitigación, se le exigió a Colombia la vigilancia de los cultivos cada dos semanas, a lo que este país solicitó que se hiciera cada mes acorde con el ciclo del insecto, evitando la duplicación de costos y haciendo efectivo el principio de equivalencia del Acuerdo de Medidas Fitosanitarias de la Organización Mundial del Comercio (OMC) (OMC, 2017, Artículo 4), en la que un país aplica a otro la medida menos restrictiva de acceso al mercado, como el caso con Perú y México según el riesgo equivalente que se esté estudiando.

De acuerdo con estos estudios, se identificaron tres insectos con alto riesgo, de una lista de 120 a 140 organismos (Millan y Forero, 2017). Estas 
especies de insectos van a la semilla, se adhieren a la fruta y pueden llegar a territorio estadounidense con el potencial de afectar allí la producción agrícola. Identificado el riesgo, se establecieron las medidas de mitigación registradas en el Systems Approach del Proposed Rule (APHIS, 2016b). El Systems Approach refiere a las medidas aplicadas para prevenir los riesgos de estos tres organismos, desde la siembra del producto hasta el punto de cosecha; así como el manejo de sanidad en los huertos, control de frutos caídos, determinación del área buffer (controlada) alrededor del cultivo. La vigilancia cada mes y la sumatoria de estas medidas hacen que se garantice un riesgo mínimo de ingreso de plagas al país de destino, en este caso, Estados Unidos.

Del año 2012 al 2017 hubo grandes avances en esta materia, siendo los años 2016 y 2017 en donde se le demostró a Estados Unidos, con documentación técnica y científica, el buen manejo que los productores de aguacate Hass lograron, tras seguir la metodología homologable y requerida por las autoridades sanitarias del país norteamericano.

Tanto la evaluación como el manejo de riesgo fueron puestos en una regla que a su vez tuvo dos etapas: el Proposed Rule (APHIS, 2016b) y el Final Rule (APHIS, 2016a). El Proposed Rule, logrado durante la administración del presidente Obama y puesto a comentario público por un periodo de 30 días, coincide con la llegada de la administración Trump, lo cual alargó el periodo dos veces, de forma que la regla salió en octubre de 2016, pero se publicó en febrero de 2017.

\subsection{Regla final}

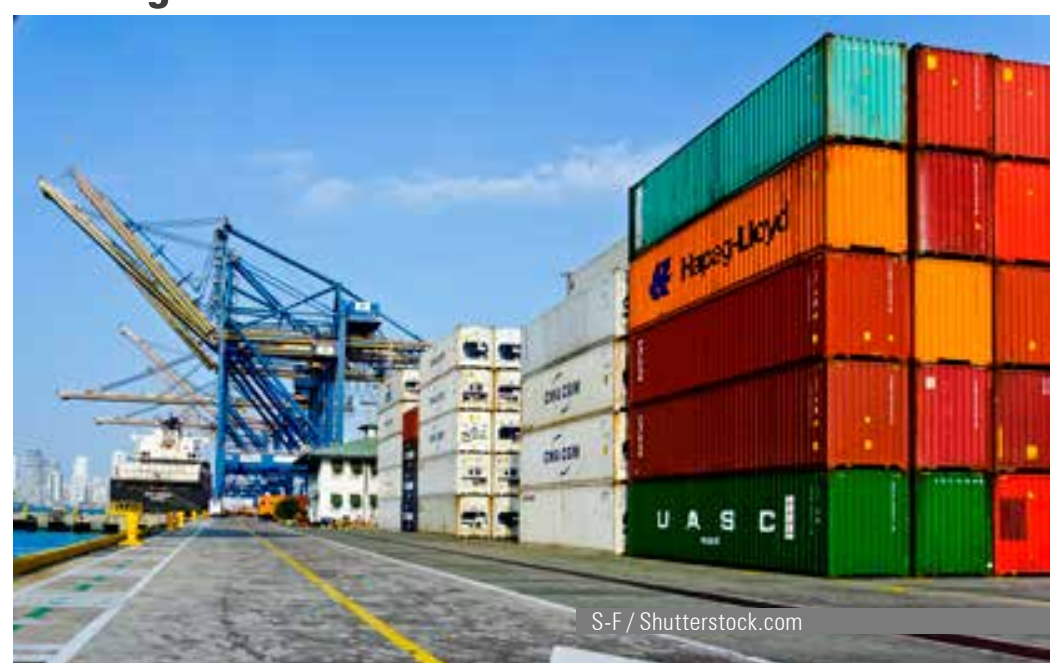

El Departamento de Agricultura de Estados Unidos, a través del APHIS, publicó la Regla Final que permite la importación de aguacate Hass en fres$\mathrm{co}$, desde Colombia hacia territorio continental de Estados Unidos, por medio de sistemas que aseguren el control de plagas dentro de su territorio. El sistema de control consta de una serie de medidas tomadas por cultivadores, empacadores y transportistas, que en conjunto minimizan el riesgo de contagio previo a la llegada al país norteamericano. Así, este sistema no se limita a la certificación, inspección y control de pestes en parcelas y empacadoras, sino también a la inspección en los puertos de entrada. (USDA, 2017).

Con la visita a Colombia del vicepresidente de Estados Unidos Mike Pence, el 14 agosto de 2017, se publicó la Regla Final con la explicación de las medidas de mitigación y manejo del riesgo. Después de esta publicación pasaron 30 días para que esta Regla Final se hiciera efectiva y en donde se dio respues- 
ta a los comentarios públicos, quedando ya establecida de manera definitiva y sujeta a evaluaciones individuales en caso de un nuevo riesgo sanitario.

A pesar de que hay un interés desde comienzos de la década de 1990 por ingresar con este producto, solo desde el intercambio de cartas en 2005, entre el ICA y el APHIS, se hace oficial. Con los dos procesos de la Regla Final, unido al lobby técnico, político y posteriormente comercial, todos los actores, importadores, personal de puertos e inversionistas dan apoyo favorable a la propuesta. La Regla Final contó con 45 comentarios, 38 de estos a favor y solo 7 en contra (Millan y Forero, 2017).

\subsection{Fase operativa}

El 14 de septiembre de 2017 empieza, entonces, la etapa operativa, con la implementación de la vigilancia sanitaria. Desde marzo de 2017 se había tenido una reunión bilateral con el APHIS en Medellín (Antioquia), para la observación de cultivos de exportación, en la cual se concretó el Plan Operativo de Trabajo, firmado el 16 de agosto de 2017 (Minagricultura, 2017). Una vez la Regla Final quedó vigente, Colombia debió informar al APHIS para el primer envío de aguacate.

A principios de noviembre, solo dos meses después de que se presentara la Regla Final, ingresaron los primeros aguacates colombianos a Estados Unidos con muy buenos resultados, como lo declara Enrique MiIlán, Director de la Oficina Comercial del Gobierno de Colombia en Estados Unidos: "La inspección técnica fue un éxito, el contenedor llegó al puerto de Port Everglades en la Florida, a esta hora hace trasbordo terrestre hacia Atlanta. Los exportadores son Hasspacol y Cartama y el comprador es Mission Produce" (2017).

\section{Compromiso público-privado}

En este propósito se involucraron principalmente tres partes: el ICA como árbitro, su homólogo APHIS y CorpoHass, este último adquiere el compromiso en la implementación de medidas y el aseguramiento de financiación a través de iniciativas como el Programa de Transformación Productiva apoyado por el Ministerio de Comercio, Industria y Turismo y Analdex, entidad que ha aportado cerca de USD 2 millones para mejorar la vigilancia sanitaria en huertos de producción, identificación, vigilancia, control y manejo del riesgo.

CorpoHass, creada en 2013, hasta el momento se ha centrado en el mercado europeo; sin embargo, su creación desde un inicio tuvo el objetivo de expandir el producto a otros mercados, con especial interés en obtener la admisibilidad en el mercado de Estados Unidos, y así lo contempla su plan de gestión estratégico 2016-2019 de donde se desprende el reto de organizar el Congreso Mundial de Aguacate Hass en Medellín para 2019.

Al propósito de llevar el aguacate Hass colombiano al mercado estadounidense se une la Corporación Colombia Internacional (CCI), una iniciativa público-privada creada en 1992 para apoyar al sector agropecuario en la implementación de procesos productivos asociativos a través de los Modelos Agro empresariales, Competitivos, Sostenibles (MACS) con metodología y marca registrada, lo que ha logrado fortalecer el agronegocio previniendo, además, la expansión de cultivos ilícitos. La Corporación ha logrado también dar impulso al clúster del aguacate Hass, incrementando las áreas sembradas y promoviendo la implementación de buenas prácticas y certificaciones internacionales de calidad, lo cual potencializa la oportunidad de acceder a mercados internacionales:

La entidad cuenta con un equipo que hace parte del Grupo Consultivo de Becarios Cochran para la implementación de la Ley de Modernización de Inocuidad Alimentaria, Food Safety Modernization Act (FSMA, por sus siglas 
en inglés), la cual será fundamental en su cumplimiento para iniciar sus exportaciones a Estados Unidos (CCl, 2017).

En la evaluación de predios se llevó a cabo la identificación de zonas, lo que dio como resultado que las más preparadas para la exportación resultaron ser Caldas y Risaralda; aunque son predios pequeños, desde el inicio de sus cultivos se implementaron medidas sanitarias más juiciosas. Antioquia, a pesar de que cuenta con un mayor número de cultivos, estos han crecido de una manera menos ordenada respecto al control de plagas. Solo unos pocos cultivos, especialmente en el sector de El Retiro (uno de los productores más grandes), han logrado erradicar los riesgos. A pesar de que este departamento alberga entre 70 a $80 \%$ de la producción nacional, no es la zona sanitariamente más preparada.

De los 90 productos colombianos que cuentan con admisibilidad, solo el aguacate Hass ha pasado por todas las etapas de manera estricta, los otros han contado con ayuda interna; de manera que el aguacate abre un importante camino para otros productos como los cítricos, los pimientos y las pasifloras.

Luis Ernesto Forero, representante de ProColombia en Washington D.C. para los asuntos relacionados con el agro, argumenta que contrario a lo que se piensa, Colombia no es un país exportador de fruta, a excepción del banano; no alcanzamos volúmenes o variedad importantes. El país hasta ahora entra en este negocio y se busca que el aguacate sea un actor importante. De igual forma, la experiencia colombiana con las frutas ha sido principalmente con la Unión Europea, en donde los requisitos de entrada son muy diferentes a los de Estados Unidos y donde hay que cumplir con cláusulas de cumplimiento, calidad y condiciones regulatorias claras.

Es de resaltar que Colombia es el segundo socio comercial en América Latina a nivel agrícola de Estados Unidos. Aun cuando México y Perú jueguen un papel tan importante en el abastecimiento del aguacate Hass hacia Estados Unidos, el potencial del mercado es tan grande que difícilmente estos países ven la incursión de Colombia como una amenaza. Igualmente, se destaca la importancia de la justificación técnica para el Gobierno de Estados Unidos, siendo el aspecto políico también relevante, así como la coyuntura con el proceso de modernización del NAFTA con México; sin embargo, de no haberse adelantado una juiciosa fase técnico-científica, difícilmente se hubiese logrado la admisibilidad para el aguacate Hass colombiano.

\section{Oportunidades para el aguacate Hass colombiano en el Este de Asia}

La noticia de la admisibilidad del aguacate Hass colombiano al mercado estadounidense es de una importancia sin igual para un país de base agrícola como Colombia. Esta noticia no solo concede la oportunidad de vender al mercado más importante del mundo, sino que también genera las bases para ser aceptado por otros mercados con potencial de crecimiento, como los del Este de Asia.

Una vez conocida la noticia, Colombia empezó a recibir delegaciones de gobierno y empresarios de varias regiones asiáticas. Una de las primeras fue la delegación de la autoridad sanitaria de China, que visitó en octubre de 2017 algunos cultivos de aguacate Hass del eje cafetero (Eje 21, 2017). China importaba 15.000 toneladas de aguacate en el 2016, y pasó a importar 32 mil en el 2017 (Trade Map, 2019) y según datos de las Naciones Unidas, es un mercado que hoy vale casi mil millones de dólares (Freshplaza, 2017).

Por otro lado, el aguacate Hass colombiano podría rápidamente participar en el abastecimiento de la creciente demanda y buenos precios en el Japón, aprovechando también momentos en los que, por variables de las cosechas y otros factores, el aguacate de México y Estados Unidos les llega de manera 
insuficiente. Con Japón se viene además trabajando en el proceso de admisibilidad del producto colombiano, pues en marzo de 2018 el Ministerio de Agricultura, Forestales y Pescadería de Japón, MAFF, por sus siglas en inglés, aceptó el protocolo fitosanitario propuesto por el ICA (Instituto Colombiano Agropecuario, 2018) y se está a la espera de la regla final.

Con este camino recorrido, el aguacate Hass colombiano podrá afianzar también procesos de admisibilidad en países del ASEAN y con Corea del Sur, el cual también viene mostrando crecimiento en el consumo y con el que ya Colombia tiene firmado un tratado de libre comercio desde hace unos años, que le permitirá acceso de 0\% en arancel a partir del año 2020. Precisamente la importancia de los mercados de Corea del Sur y Japón se ve reflejada en las cifras que representan al día de hoy y sus proyecciones al futuro.

El gobierno nacional a través de entidades como ProColombia y el Programa de Transformación Productiva han iniciado estrategias de promoción para fortalecer la oferta exportadora dando un lugar primordial a las frutas, dirigido a países con los que ya se tiene un acuerdo comercial vigente, como es el caso de Corea del sur, país altamente importador de productos agrícolas. Este propósito, a su vez, busca extenderse a otros países asiáticos como Japón, con quien ya se tiene en curso la negociación del Acuerdo de Asociación Económica como parte de la estrategia comercial en Asia.

Del total de las exportaciones de Colombia hacia países con los que tiene ya acuerdos comerciales vigentes, el porcentaje de participación de exportaciones hacia Corea de Sur fue de 1,5\%, con una suma de 457 millones de dólares (en valor FOB) para 2017, y de 627 millones de dólares para 2018, lo que representó una variación positiva de 37,3\% (Mincit, 2019). Para el caso de Japón, Colombia exportó productos por un valor de 557 millones de dólares (valor FOB) para 2017, cifra que disminuyó en 2018 a 474 millones de dólares. (DANE, 2019).

En el sector agroindustrial, las exportaciones de Colombia hacia Corea del Sur registraron para 2017 una cifra de 9 millones de dólares (en valor FOB) y para 2018, de 8 millones de dólares, reflejando una variación porcentual de $-5,9 \%$. Dentro de los principales productos agroindustriales exportados a Corea del Sur se destacan café, con una cifra de 83 millones de dólares y una variación negativa de $7 \%$ respecto al año anterior; flores y capullos, con una cifra de 4 millones y medio de dólares y una variación positiva de 4,6\% respecto al año anterior; extractos y concentrados de café, té o yerba mate, con una cifra de 6 millones de dólares, y una variación negativa de 3,4\% respecto al año anterior y cacao en polvo sin adición de azúcar ni otro edulcorante, con una cifra de 86 mil dólares, y una variación positiva de 11,6\% respecto a 2017. Cabe destacar que Antioquia se ubica en segundo lugar, después del Atlántico, como uno de los principales departamentos de Colombia que exportan hacia este país asiático. (Mincit, 2019)

Para el caso de Japón, Colombia exportó café por un valor de 195 millones de dólares en 2018; en flores, un valor de 53 millones de dólares; en extractos y concentrados de café, té o yerba mate, un valor de 12 millones de dólares y en el caso del cacao, un valor de 18 mil dólares para este mismo año. Se destaca que, dentro de este grupo de productos en mención, solo el cacao reportó un crecimiento considerable de 55\% entre los años 2014 a 2018 (Trademap, 2019).

Es precisamente por las cifras reportadas y por la creciente cercanía con dichos países desde Colombia, que se pude vislumbrar el potencial de exportación que una fruta como el aguacate Hass tendrá en un futuro cercano. 


\section{Discusión: los retos que enfrenta el aguacate Hass colombiano}

El caso nos muestra la manera como se abren las puertas a un mercado más grande para nuestro país, generando también mayor atracción de inversión extranjera y nuevas alianzas comerciales; se dinamiza el sector desde todos sus ángulos y los colombianos tienen nuevas esperanzas a nivel económico y social.

Sin dejar de aplaudir la rápida y efectiva reacción de los productores y comercializadores colombianos, vale la pena reflexionar sobre los siguientes aspectos, los cuales pueden ir en contravía de un buen desempeño a futuro:

\subsection{Las plagas}

Colombia posee características geográficas que tienen muy pocos países en el mundo; es un país tropical, pero a la vez con páramos y variados pisos térmicos que brindan un potencial de cultivo sin igual para el aguacate Hass, contando a su vez, con la herencia cafetera que dota a los trabajadores de dichas zonas con la experiencia para manejar este tipo de cultivos. Sin embargo, esta misma característica hace que se deban tener elevados controles a nivel de plagas, los cuales, si no se logran manejar adecuadamente, podrían perjudicar la calidad en el largo plazo, según afirma en entrevista José Alberto Vélez, presidente de CorpoHass (CorpoHass, 2017). Este aspecto es, por ejemplo, de vital importancia también para la entrada del aguacate Hass colombiano al mercado japonés, dónde el mayor énfasis está en el control de pestes asociadas a productos agrícolas (Muñoz, 2018).

\subsection{La asociatividad}

Es importante resaltar este tema. Es clave actuar en equipo y también ser capaces de consolidar un gremio sólido de productores y comercializadores del aguacate Hass, que pueda representar bien sus intereses, prender las alarmas y propiciar la asesoría necesaria para el beneficio conjunto. La adecuada asociatividad ayuda además a velar por el aprovechamiento de las ventanas de oportunidad. Los períodos de producción y envío en relación con competidores como México, con los que existen períodos de cosecha muy similares, se pueden manejar a nivel agronómico en huertos de producción, podas y manejos de clima, para retrasar la producción y de esta manera cubrir períodos de desabastecimiento del producto.

\subsection{La calidad}

El United States of America: Avocados Market Report, prevé que para 2025 el volumen del mercado del aguacate en Estados Unidos llegue a 1,3 millones de toneladas, partiendo de un crecimiento sostenido de 3,2\% desde 2016 (IndexBox, 2017). Debido a que el consumo del aguacate Hass crece de manera mucho más elevada que la producción a nivel mundial, podría entenderse que cualquier calidad sería bien apreciada; sin embargo, es importante continuar el esfuerzo de producción de calidades óptimas, de manera que la reputación del producto colombiano no sea la de un aguacate de mediano nivel, como lo consideró el señor Giovanni Gómez, director de asuntos económicos de Analdex, quien, en entrevista, comentó que según informes de calidad internacional, el producto colombiano obtiene una calificación de 5-6 en una escala de 1 a 10 (Giovanni Gómez, 2017). Dicho factor también podría repercutir en el nivel de precios al detal del producto, que para 2016 fluctuaron entre USD 1,32 y USD 0,95 por unidad en Estados Unidos (IndexBox, 2017). 


\section{Referencias bibliográficas}

- $\quad$ Agriculture, U. S. D. of. (s.f.). APHIS, Animal and Plant Health Inspection Service.

- Agronet. (2015). Exportaciones colombianas de aguacate Hass triunfan en Europa. Recuperado de http:// www.agronet.gov.co/noticias/Paginas/Noticia1471.aspx

- Gómez Tagle, A. (2018). Desafíos sociales, ambientales y a la salud del oro verde de América Latina. Conferencia en Universidad Eafit, octubre 5 de 2018.

- AmCham Colombia. (2015). Una oportunidad cercana para los exportadores colombianos. Recuperado de https://amchamcolombia.wordpress.com/2015/09/25/aguacate-Hass-una-oportunidad-cercana-para-losexportadores-colombianos/

- Analdex. (2016). Informe Aguacate 2016. Recuperado de: http://www.analdex.org/2016/06/20/informeaguacate-2016/. Consultado el 17 de octubre de 2017

- Analdex. (2017). Los retos del aguacate Hass colombiano en los mercados internacionales (pp. 1-29). ANALDEX.

- Animal and Plant Health Inspection Service (APHIS). (2016a). Final Regulatory Flexibility Analysis: Hass Avocado Imports from Colombia into the Continental United States.

- Animal and Plant Health Inspection Service (APHIS). (2016b). Imports: Hass Avocados from Colombia.

- Animal and Plant Health Inspection Service (APHIS). (2010). Importation of Hass Avocados from Peru. Federal Register Nol. 75, No. 1 /Monday, January 4, 2010 /Rules and Regulations.

- Asociación Hortifrutícola de Colombia. (2017). Asohofrucol ejecutará Convenio de Admisibilidad para aguacate Hass en Estados Unidos.

- Asohofrucol. (s.f.). Asohofrucol. Recuperado de: http://www.asohofrucol.com.co/. Consultado el 17 de octubre de 2017

- Bredahl, M. E. (2001). Trade liberalization Under NAFTA: trade in avocados (No. 751-2016-51435, p. 218).

- Pavas, C.A. (2015). Organización de cadena productiva del aguacate [Presentación de Power Point, disponible en línea]. Ministerio de Agricultura, 1-20. Recuperado de: https://docplayer.es/31688338Organizacion-de-cadena-productiva-del-aguacate-octubre-de-2015-cesar-andres-pavas-tabaressecretario-tecnico-nacional.html

- Candela, C. (2017). Entrevista con Claudia Candela, ProBogotá, octubre 25 de 2017. Profesoras María Alexandra Guerra y Carolina Ardila, Universidad EAFIT.

- Comité de paltas Hass de Chile. (2015). Comité de paltas Hass de Chile. Recuperado de: www.paltahass. cl/esp/palta-Hass. Consultado el 4 de octubre de 2017.

- Comité de paltas Hass de Chile. (2018). Estadísticas. Recuperado de: http://www.paltahass.cl/esp/ exportacion-por-destino. Consultado el 25 de enero de 2018.

- CorpoHass (2017). Entrevista con José Alberto Vélez, Presidente, y Jorge Restrepo, Director Ejecutivo de CorpoHass, octubre 30 de 2017. Profesoras María Alexandra Guerra y Carolina Ardila, Universidad EAFIT.

- Corpoica (2017). "Trabajamos para que el aguacate Hass, se convierta en el cuarto producto agrícola colombiano de exportación". Recuperado de: http://www.corpoica.org.co/noticias/generales/sgraguacate-antioquia/. Consultado el $1^{\circ}$ de octubre de 2017.

- Corporación Colombia Internacional - CCI (noticias). (2017). "Más y más oportunidades para los pequeños productores de aguacate Hass en el mercado de Estados Unidos". Recuperado de: http://www.cci.org. co/mas-y-mas-oportunidades-para-los-pequenos-productores-de-aguacate-hass-en-el-mercado-deestados-unidos $/ \% 0 \mathrm{~A} \% 0 \mathrm{~A}$.

- Department of Agriculture (APHIS) Animal and Plant Health Inspection Service. Importation of Hass Avocados from Peru. Federal Register Nol. 75, No. 1 /Monday, January 4, 2010 /Rules and Regulations.

- Departamento Administrativo Nacional de Estadística - DANE (2019). Colombia, principales destinos de las exportaciones. 2008p - 2019p (febrero). [Excel] Recuperado de: https://www.dane.gov.co/index.php/ estadisticas-por-tema/comercio-internacional/exportaciones. Consultado el 15 de marzo 2019.

- Dinero (2017). Aguacate: el oro verde de la economía colombiana. Recuperado de: http://www.dinero. com/edicion-impresa/informe-especial/articulo/aguacate-exportacion-y-mercado-en-colombia/243434. Consultado el 30 de septiembre de 2017.

- Eje 21. (2017). Delegación china visitará plantaciones de aguacate Hass en el Eje Cafetero. Recuperado de: http://www.eje21.com.co/2017/10/delegacion-china-visitara-plantaciones-de-aguacate-hass-en-el-ejecafetero/. Consultado el 17 de noviembre de 2017.

- El Financiero (2017a). 3 gráficas para entender la importancia del aguacate para México. Recuperado de: http://www.elfinanciero.com.mx/rankings/la-importancia-del-aguacate-para-mexico-en-graficas.html. Consultado el 4 de octubre de 2017.

- El Financiero (2017b). Estos cuatro países compiten con México por el mercado del aguacate. Recuperado de: http://www.elfinanciero.com.mx/economia/los-principales-exportadores-de-aguacate-del-mundo. html. Consultado el 4 de octubre de 2017. 
- Ellet, W. (2007). The case Study Handbook, How to Read, Discuss, and Write Pesuasively About cases. Harvard Business Press, 1-26. Recuperado de: https://doi.org/10.1017/CB09781107415324.004.

- Milán, E. y Forero, L.E. (2017). Entrevista con Enrique Millán y Luis Ernesto Forero, septiembre 19 de 2017. Profesoras María Alexandra Guerra y Carolina Ardila, Universidad EAFIT.

- FAOSTAT. (2016). FAOSTAT. Crops. Recuperado de: http://www.fao.org/faostat/en/\#data/QC. Consultado el 29 de julio de 2018.

- FAOSTAT. (2014). FAOSTAT. Trade - Crops and Livestock Products. Recuperado de: http://www.fao.org/ faostat/en/\#compare. Consultado el 26 de noviembre 26 de 2018.

- Freshplaza (2015). La temporada de aguacates en la República Dominicana está en marcha. Recuperado de: http://www.freshplaza.es/article/93006/La-temporada-de-aguacates-en-la-República-Dominicanaestá-en-marcha. Consultado el 4 de octubre de 2017.

- Freshplaza (2017). Australia busca proveer a China en su creciente apetito de aguacates. Recuperado de: http://www.freshplaza.es/article/109825/Australia-busca-proveer-a-China-en-su-creciente-apetito-deaguacates. Consultado el 4 de octubre de 2017.

- Gómez, G. (2017). Entrevista a Giovanni Gómez de Analdex, octubre 30 de 2017. Profesoras María Alexandra Guerra y Carolina Ardila, Universidad EAFIT.

- HAB. (2017). Hass Avocado Board.

- ICA. (s.f.). Centro de Excelencia Fitosanitaria.

- IndexBox. (2017). United States of America : Avocados - Market Report. Analysis and Forecast to 2025.

- Instituto Colombiano Agropecuario (ICA). (2013). Productores y exportadores de aguacate Hass conforman la corporación nacional.

- Instituto Colombiano Agropecuario. (2018). Colombia a un paso de exportar aguacate Hass a Japón. Recuperado de: https://www.ica.gov.co/Noticias/Colombia-exportacion-aguacatehass-Japon-ICA.aspx

- Jouanjean, M. A., Maur, J. C., \& Shepherd, B. (2016). US phytosanitary restrictions: the forgotten non-tariff barrier. Journal of International Trade Law and Policy, 15(1), 2-27.

- Minagricultura. (2016). Aguacate Hass colombiano entra en la recta final para exportar a Estados Unidos. Recuperado de: https://www.minagricultura.gov.co/noticias/Paginas/Aguacate-Hass-colombiano-entraen-la-recta-final-para-exportar-a-Estados-Unidos.aspx. Consultado el 30 de septiembre de 2017.

- Minagricultura. (2017). MinAgricultura agiliza trámite final para que dos mil productores de aguacate Hass puedan exportar a EEUU.

- Mincit. (2017). Luz verde de Estados Unidos al aguacate Hass colombiano, impulso a inversión extranjera y empleo. Recuperado de: http://www.mincit.gov.co/publicaciones/38859/uz_verde_de_estados_ unidos_al_aguacate_hass_colombiano_impulso_a_inversion_extranjera_y_empleo. Consultado el $30 \mathrm{de}$ septiembre de 2017.

- Mincit. (2019). Estos son los productos de exportación que tuvieron mayor crecimiento en 2018. Recuperado de: http://www.mincit.gov.co/prensa/noticias/comercio/productos-de-exportacion-de-mascrecimiento-2018. Consultado el 29 de enero de 2019.

- Ministerio de Comercio Industria y Turismo. (2019). Comercio exterior colombiano: Seguimiento a los acuerdos comerciales. Oficina de Estudios Económicos. [pdf] Recuperado de: http://www.mincit.gov.co/ getattachment/estudios-economicos/seguimiento-tlc/seguimiento-vision-nacional/acuerdos-comercialesvigentes/oee-lvh-seguimiento-av-ene-feb19.pdf.aspx. Consultado el febrero 22 de 2019.

- Ministerio de Relaciones Exteriores de Perú. (2011). Perfil del mercado de palta Hass en Estados Unidos. Oficina de Promoción Comercial e Inversión.

- Neederlands Ministry of Foreign Affairs. (2016). Exporting Fresh Avocados to Europe. Recuperado de: https://www.cbi.eu/market-information/fresh-fruit-vegetables/avocado/europe/. Consultado el 4 de octubre de 2017.

- Muñoz, Izasa, Stiven (2018). Opportunities and Challenges for the Colombian Hass Avocado in Countries with Admissibility Processes in Progress: The case of Japan. Master in International Business Thesis, Universidad EAFIT, Medellín, Colombia.

- OMC. (2017) "Acuerdo sobre la aplicación de medidas sanitarias y fitosanitarias". Recuperado de: https:// www.wto.org/spanish/tratop_s/sps_s/spsagr_s.htm

- Orden, D., \& Peterson, E. (2003). Sanitary and Phytosanitary Measures: The Case of Mexican Avocados Nishita Bakshi (Abstract). Evaluation, (July).

- ProHass Perú. (2017). Más paltas peruanas a pesar de El Niño. Recuperado de: http://www.prohass.com. pe/blog/2017/08/mas-paltas-peruanas-a-pesar-de-el-nino. Consultado el 4 de octubre de 2017.

- Rudert, B. (2017). Aguacate Hass iUna alternativa para la economía fronteriza? Recuperado de: www. listindiario.com/economia/2017/04/19/462367/aguacate-Hass-una-alternativa-para-la-economiafronteriza

- Simfruit. (2016). Resumen del mercado mundial de la palta. Recuperado de: http://simfruit.cl/ internacionales/2101-resumen-del-mercado-mundial-de-la-palta.html. Consultado el 4 de octubre de 2017. 
- The U.S. Department of Agriculture's (USDA). (2017). APHIS Publishes Final Rule to Allow Fresh Hass Avocado Fruit from Colombia into the Continental United States.

- Trade Map. (2019). Trade Statistics for International Business Development. Recuperado de: https://www. trademap.org/lndex.aspx.

- United States Department of Agriculture. (2017). A Pathway-Initiated Risk Assessment.

- USDA. (2017). Animal and Plant Health Inspection Service. Recuperado de: https://epermits.aphis.usda. gov/manual/index.cfm?NEW=1\&COMMOD_ID=12\&ACTION=commSummCountryP Consultado el 17 de octubre de 2017. 